\title{
A Faster Path to Solar Water Splitting
}

Finding new wide-bandgap light absorbers that are stable in aqueous solutions is a long-standing challenge in photoelectrochemical water splitting research. Two papers in this issue describe recent advances in high throughput experimentation that may accelerate the discovery of suitable materials.

Roel van de $\mathrm{Krol}^{1,2, *}$

${ }^{1}$ Institute for Solar Fuels, Helmholtz-Zentrum Berlin für Materialien und Energie GmbH, Hahn-Meitner-

Platz 1, 14109 Berlin, Germany

${ }^{2}$ Department of Chemistry, Technical University of Berlin, Straße des 17. Juni 124, 10623 Berlin, Germany

*Correspondence: roel.vandekrol@helmholtz-berlin.de

Semiconducting materials that are able to absorb sunlight and use this energy to directly drive an electrochemical reaction at their surface have fascinated researchers for decades. Most photoelectrochemical (PEC) studies have focused on water splitting, a thermodynamically up-hill reaction that could offer an attractive pathway for the long-term capture and storage of solar energy by producing 'green' hydrogen. Other useful PEC reactions are the oxidation of harmful pollutants or even the reduction of $\mathrm{CO}_{2}$ to carbon monoxide or hydrocarbons. To drive the electrochemical reaction of interest, photovoltages of at least $1.5 \mathrm{~V}$ are needed. Such high voltages require tandem systems in which at least two absorbers are connected in series. While silicon is usually considered as the optimal bottom absorber, finding an efficient and chemically stable top absorber with a bandgap of around 1.5 $-2.0 \mathrm{eV}$ has been a long-standing and largely unsolved challenge [1].

The search for suitable photoanode materials has mostly concentrated on metal oxides, one the few materials classes that shows good chemical stability under the highly oxidizing conditions that enable water splitting. Metal oxides are easy to synthesize and process compared to classic semiconductors like $\mathrm{Si}$ and GaAs, but those that absorb visible light tend to suffer from poor charge transport properties due to the formation of polarons, excitons, or defects. The good news is that only a tiny fraction of the $\sim 19,000$ possible ternary oxides and $>200,000$ quaternary oxides has been studied thus far [2], which makes it likely that the best materials are still waiting to be discovered. The bad news is that there are currently no robust and proven strategies to identify promising candidates. The calculation of polaronic and midgap defect states is still too computationally expensive for in silico materials design with high throughput electronic structure calculations. As a result, the search for novel photoelectrode materials has thus far mostly relied on chemical intuition and serendipitous discoveries.

Two papers in this issue report advances in the discovery and optimization of metal oxide photoanodes using high throughput experimentation. Both papers are from the high throughput team at the Joint Center for Artificial Photosynthesis (JCAP) in the USA. In the first paper, Zhou et al. have studied a family of copper vanadate-based quaternary compositions, Cu-V-M-O, where $\mathrm{M}=\mathrm{Mg}$, $\mathrm{Ca}$, $\mathrm{Sr}$, or Fe [3]. Copper vanadates (CVOs) have attracted interest because they exhibit narrow bandgaps as well as good photochemical stability, a combination that is relatively rare for $n$-type semiconductors. Thin film gradient composition libraries were made with reactive co-sputtering and the structural, optical, and photoelectrochemical properties of many hundreds of distinct compositions were measured. Especially noteworthy are the extensive photoelectrochemical measurements; using a scanning droplet cell, no less than four chopped-light voltammograms, each with a different illumination wavelength, were measured for each of the 840 samples in the library. This dataset is one of the largest ever made in PEC studies and allowed the authors to identify several general trends. One is that alloying with divalent alkaline earth elements on the $\mathrm{Cu}$ sites improves the PEC properties of CVOs, and another is that V-rich compositions 
showed relatively high photocurrents at low photon energies. Furthermore, a large spread in the generated photovoltage was found for samples that show the highest photocurrents. This is important because while most PEC studies tend to emphasize photocurrent as a performance metric, it is the internally generated photovoltage that determines the available driving force for the electrochemical reactions. Sr-alloyed $\mathrm{Cu}_{5} \mathrm{~V}_{2} \mathrm{O}_{10}$ showed the highest performance in this study, and six promising new quaternary compositions were identified that warrant follow-up studies.

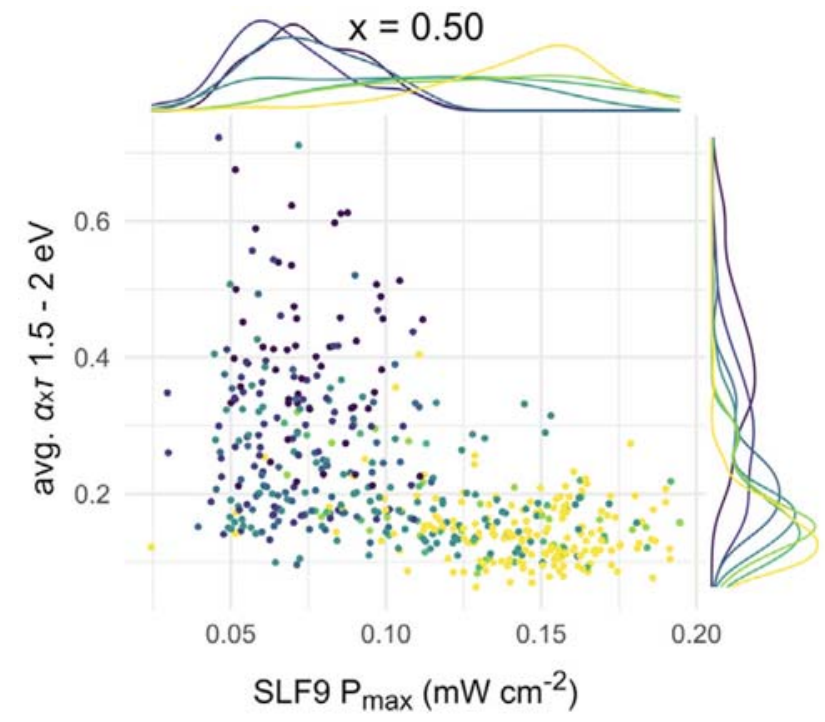

Figure 1. Scatter plot of the average optical absorption of co-alloyed $\mathrm{Cu}_{2} \mathrm{~V}_{2} \mathrm{O}_{7}$ as a function of the photoelectrochemical power $\left(\boldsymbol{P}_{\max }\right)$ produced by the material.

The color of the points indicates the alloy loading, ranging from $0 \%$ (black) to $8 \%$ (yellow). Alloying increases $\mathrm{P}_{\max }$, but reduces the optical absorption at low photon energies (reproduced with permission from Newhouse et al. [4]).

In the second paper, the JCAP team switched from high throughput photoanode discovery to high throughput modification of a single photoanode material, $\beta-\mathrm{Cu}_{2} \mathrm{~V}_{2} \mathrm{O}_{7}[4] . \mathrm{Cu}_{2} \mathrm{~V}_{2} \mathrm{O}_{7}$ shows good visible light absorption starting at photon energies of $2 \mathrm{eV}$, but this does not result in good photoactivity at lower energies due to the formation of excitons [5]. Newhouse et al. attempted to disrupt the formation of excitons by combinatorial (co)doping (or rather alloying) of $\beta-\mathrm{Cu}_{2} \mathrm{~V}_{2} \mathrm{O}_{7}$. Using inkjet printing, they fabricated combinatorial libraries of $\mathrm{Cu}$-rich, $\mathrm{Cu}$-poor, and stoichiometric $\mathrm{Cu}_{2} \mathrm{~V}_{2} \mathrm{O}_{7}$ that were co-alloyed with six elements ( $\mathrm{Ca}, \mathrm{Gd}, \mathrm{Hf}, \mathrm{La}, \mathrm{Lu}, \mathrm{Zr}$ ) and their pairwise combinations. With concentrations ranging between 0 and 7.3\%, more than 1800 different compositions were synthesized. They found that $\mathrm{Ca}$ appears to enhance to oxygen evolution reaction and that the combination of $\mathrm{Ca}$ with either $\mathrm{Hf}, \mathrm{Zr}$, or $\mathrm{La}$ leads to a 2.7-fold increase in photoactivity. One subtle, yet intriguing finding is that the optimal ratio of co-alloying elements, i.e., $z$ in $\mathrm{Cu}_{1-x} \mathrm{~V}_{x} \mathrm{O}_{\delta}:\left(\mathrm{Ca}_{1-\mathrm{z}} \mathrm{B}_{z}\right)_{\mathrm{y}}$, where $\mathrm{B}$ is $\mathrm{La}, \mathrm{Hf}$, or $\mathrm{Zr}$, shifts to higher values if the base composition becomes increasingly $\mathrm{Cu}$-poor (i.e., at higher values of $x$ ). This trend would have been nearly impossible to identify in a regular PEC study and illustrates the power of the high throughput approach. The latter is even more clearly illustrated by Fig. 1, which reveals that while it is indeed possible to improve the transport properties (evidenced by the increase in generated power, $\mathrm{P}_{\max }$ ), it comes at the cost of reduced optical absorption at lower photon energies.

The first combinatorial studies on photoelectrode materials date back to 2002 with the work of McFarland et al. [6], followed a few years later by Parkinson ([2] and refs. therein) and several other groups. Most of these early studies report modifications of established photoelectrode materials and focus mainly on 
establishing high throughput methods based on electrodeposition or variations of ink jet printing. Later efforts by e.g. Ludwig et al. [7] extended this to vacuum-based deposition techniques, such as sputtering. One of the first examples of a truly new photoelectrode material was p-type $\mathrm{CuBi}_{2} \mathrm{O}_{4}$, a $1.8 \mathrm{eV}$ bandgap material reported by the group of Sayama [8]. The work of the JCAP team, headed by John Gregoire, probably represents the largest concerted effort in the field. Over the past decade, they have built up an impressive infrastructure for high throughput synthesis and characterization of photoelectrodes as well as electrocatalysts.

High throughput studies are not only demanding in terms of the required research infrastructure, the design of such studies and the analysis of the data also comes with many challenges. Every high throughput experiment needs a good starting point, and the JCAP team have established an effective research pipeline in which promising starting points are provided by high throughput theory [9]. To convert large datasets into useful insights, a judicious choice of performance indicators is needed. Takeuchi already warned that combinatorial techniques are not suitable for every application [10], and it should be realized that photoelectrodes are not the easiest candidates due to the strong influence of processing conditions on the performance. Incomplete crystallization, small amounts of defects, and the presence of grain boundaries can easily lead to false negatives. Conversely, the relatively low photoactivity of most oxides can lead to false positives, since it often easy to improve a poorly performing material by making it slightly less poor. The chance of a false positive will of course decrease as the baseline performance of the library improves.

The large amount of resources (time, money, personnel) that have to be invested in an effective high throughput infrastructure means that such studies are unlikely to be verified or reproduced in their entirety by other groups, as is common for other types of PEC studies. Instead, high throughput studies contribute by offering concrete suggestions for promising new compositions, such as the Sr-alloyed $\mathrm{Cu}_{5} \mathrm{~V}_{2} \mathrm{O}_{10}$ and the additional six new quaternary compositions proposed by Zhou et al. [3]. They also contribute by revealing subtle trends that are normally obscured by the relatively large sample-to-sample variations encountered in typical PEC studies. Such trends may offer interesting avenues for further exploration, or even provide direct support for theoretical predictions (like the role of $\mathrm{Cu} 3 \mathrm{~d}$ states in CVO [3]). Last but not least, high throughput studies can tell us what does not work. An excellent example of this is Figure 1 , which shows that $\beta-\mathrm{Cu}_{2} \mathrm{~V}_{2} \mathrm{O}_{7}$ is unlikely to show high energy conversion efficiencies at low photon energies [5]. The importance of reporting negative results cannot be overstated; the PEC community can no longer afford to spend decades on a single material, like we did with $\mathrm{TiO}_{2}$. With so many candidate materials and so little time left to transform our energy infrastructure, it is important to 'fail quickly' [1]. The recent developments in high throughput experimentation, exemplified by the two papers from the JCAP team, offer a promising path to also succeed quickly.

1. van de Krol, R. and B.A. Parkinson (2017). Perspectives on the photoelectrochemical storage of solar energy. MRS Energy \& Sustainability 4, E13.

2. Woodhouse, M. and B.A. Parkinson (2009). Combinatorial approaches for the identification and optimization of oxide semiconductors for efficient solar photoelectrolysis. Chem. Soc. Rev. 38, 197210.

3. Zhou, L., et al. (2020). Quaternary Oxide Photoanode Discovery Improves the Spectral Response and Photovoltage of Copper Vanadates. Matter, this issue.

4. Newhouse, P.F., et al. (2020). Enhanced Bulk Transport in Copper Vanadate Photoanodes Identified by Combinatorial Alloying. Matter, this issue.

5. Wiktor, J., et al. (2018). Sizable Excitonic Effects Undermining the Photocatalytic Efficiency of beta$\mathrm{Cu}_{2} \mathrm{~V}_{2} \mathrm{O}_{7}$. J. Phys. Chem. Lett. 9, 5698-5703.

6. Baeck, S.H., et al. (2002). Combinatorial electrochemical synthesis and characterization of tungstenbased mixed-metal oxides. J. Comb. Chem. 4, 563-568. 
7. Vidyarthi, V.S., et al. (2011). Enhanced photoelectrochemical properties of $\mathrm{WO}_{3}$ thin films fabricated by reactive magnetron sputtering. Int. J. Hydrogen Energy 36, 4724-4731.

8. Arai, T., et al. (2007). High-throughput screening using porous photoelectrode for the development of visible-light-responsive semiconductors. J. Comb. Chem. 9, 574-581.

9. Yan, Q.M., et al. (2017). Solar fuels photoanode materials discovery by integrating high-throughput theory and experiment. Proc. Natl. Acad. Sci. 114, 3040-3043.

10. Koinuma, H. and I. Takeuchi (2004). Combinatorial solid-state chemistry of inorganic materials. Nat. Mater. 3, 429-438. 